\title{
Flow-law hypotheses for ice-sheet modeling
}

\author{
Richard B. Alley \\ Earth System Science Center and Department of Geosciences, The Pennsylvania State University, \\ University Park, Pennsylvania 16802, U.S.A.
}

\begin{abstract}
Ice-flow modeling requires a flow law relating strain rates to stresses in situ, but a flow law cannot be measured directly in ice sheets. Microscopic processes such as dislocation glide and boundary diffusion control both the flow law for ice and the development of physical properties such as grain-size and $c$-axis fabric. These microscopic processes can be inferred from observations of the physical properties, and the flow law can then be estimated from the microscopic processes.

A review of available literature shows that this approach can be imperfectly successful. Interior regions of large ice sheets probably have depth-varying flow-law "constants", with the stress exponent, $n$, for power-law creep less than 3 in upper regions and equal to 3 only in deep ice; $n$ probably equals 3 through most of the thickness of ice shelves and ice streams.
\end{abstract}

\section{INTRODUGTION}

The flow law relating strain rate to stress, temperature and ice properties is to glaciology what the Holy Grail was to chivalry - an important goal but one that may not be attainable. As Lliboutry and Duval noted (1985, p. 219), "any attempt to determine the creep law of polycrystalline ice is illusory". This is because various processes always compete in deformation: dislocation glide, dislocation climb, boundary diffusion, bulk diffusion, etc., and their importance varies within the range of natural environments. The "flow law" either must include all of these processes explicitly, becoming unmanageably complicated, or it must change as the dominant processes change.

It nonetheless is true that we need some sort of flow law to conduct ice-flow modeling for ice-core dating and studies of ice-sheet evolution. Furthermore, it is true that the large ice sheets of Greenland, East Antarctica and West Antarctica, and some cold small ice sheets and mountain glaciers, show consistent patterns of physical behavior. For example, most ice on Earth forms as firn and densifies without melting, undergoes normal stresses (usually vertical compression and longitudinal extension) in upper zones of ice sheets, switches to basal shear deeper and eventually switches back to normal stresses in ice streams and ice shelves. It is possible that one could write different expressions relating stresses and strain rates for different regimes, and in fact this is done today (e.g. iceflow modelers often use a different activation energy for creep above and below $-10^{\circ} \mathrm{C}$, and often use different enhancement factors for Wisconsinan ice compared to Holocene ice in Greenland and Arctic Canada). Such an expression is an approximation of "the" flow law, and properly should be referred to as such or as "a" flow law; commonly, however, glaciologists have referred to such an approximation as "the" flow law.

Approaches to approximating the flow law within any one regime can include theory, laboratory experiment and field observations. All are valuable, but all suffer drawbacks as well. Theoretical studies of microscopic processes (e.g. dislocations) lead directly to flow-law parameters, but often must rely on poorly known physical quantities. Laboratory studies allow control of all relevant variables, but cannot avoid the fact that the time-scale for natural ice deformation is centuries to millennia, but heroics are needed (and have been utilized) to extend laboratory work to years. Field observations have just the opposite problem; the time-scale is correct, but the boundary conditions and in situ stresses are difficult to constrain accurately.

One possible way to minimize the problems of field studies of the flow law, as suggested by D. D. Blankenship, J. F. Bolzan and coworkers (PIREX Workshop, 1990), is to use formal geophysical inverse techniques to analyze concentrated borehole and surface observations on an ice sheet. Data on accumulation rates, ice geometry, temperature, fabric and texture would provide inputs for forward models of ice velocities (vertical and horizontal). Free parameters in the forward models would be adjusted using an extension of Newton's method to optimize the fit between the predicted and observed velocities, and the goodness-of-fit would be determined statistically. Different hypotheses of the flow law would be tested as forward models, and the favored approximation of the flow law would be that hypothesis that provides the best fit to the data. Such an experiment must start with hypotheses for the flow law, based on the available laboratory, field and theoretical information.

Ice deformation, grain growth and fabric development all arise from microscopic processes (dislocation motion, diffusion) that are difficult or impossible to 
observe directly. Of these, grain growth and fabric development are easier to observe than ice deformation. It thus seems possible to use the observations of grain growth and fabric development as a probe to infer the microscopic processes operating and, from these microscopic processes, to approximate the flow law. Here, I combine this technique with a brief review of other available information to produce hypotheses for the power-law exponent and the fabric-enhancement factor in the usual power-law approximation of the flow law. The effort is interesting, though imperfectly successful.

Unless otherwise stated, I restrict consideration to typical ice-sheet conditions: strain rates, stresses and cumulative strains within observed ranges; $-70^{\circ} \mathrm{C}<$ temperature < pressure-melting point; and typical dust and impurity levels (dirty basal ice and ice containing visible volcanic dust are special cases not considered here). I begin by presenting results of earlier research on the flow law, grain growth and fabric development. With each result, I provide a personal opinion on the level of support for that result, on a scale of $\{0\}$ to $\{5\}$, in which $\{5\}$ is virtually certain (e.g. Newtonian physics as a large-body approximation) and certainty decreases to $\{0\}$, an interesting idea with no support. Following this, I consider the energy involved in the important microscopic and macroscopic processes, and then I combine the information on deformation, grain growth, fabric and energy for different regimes in the ice sheet. This leads to hypotheses for the flow law for each regime.

\section{RESULTS - FLOW LAW}

\section{A. Ice deforms primarily by dislocation glide on the basal plane $\{4\}$}

Many mechanisms can contribute to deformation, and there almost certainly are situations in which ice enters other deformation fields. If grain-size were small enough, diffusional creep (Nabarro-Herring or Coble creep) would become important (Duval and others, 1983). In low-density firn, grain-boundary sliding may be dominant (Alley, 1987). However, for conditions thought to occur in densified, ice-sheet ice, direct laboratory observations and theory strongly support dislocation glide on the basal plane (Weertman, 1973, 1983; Duval and others, 1983; Fukuda and others, 1987). Other glide systems are 100 to 1000 times "harder" than basal glide.

The basal plane has only two independent slip systems, but continuum deformation of a polycrystal requires four or five independent slip systems. The basal glide must be accommodated in some way: by hard, prismatic glide; by dislocation climb; by grain-boundary sliding; by Nabarro-Herring type diffusion; or in some other manner. Diffusional processes (grain-boundary migration, dislocation climb, Nabarro-Herring creep) seem likely considering the low strain rates and relatively high temperatures (almost always $>70 \%$ of the absolute melting temperature, and often $>90 \%$ ) of most natural ice deformation.

B. If strain rate, temperature, stress and cumulative strain are large enough, strain rate increases approximately with the third power of the stress $\{4\}$

The (or a?) flow law for ice usually is written as

$$
\dot{\varepsilon}=A_{\mathrm{o}} \exp \left[-\frac{Q}{k T}\right] \sigma^{n} \text {. }
$$

Here $\dot{\varepsilon}$ is the strain rate, $A_{0}$ is a prefactor that may depend on such factors as fabric, grain-size or impurity concentrations (and can be written as $a A$, where $a$ is an enhancement factor on the "normal" behavior $A$, and may be a tensor quantity), $Q$ is the activation energy, $k$ is Boltzmann's constant, $T$ is the absolute temperature, $\sigma$ is the stress deviator and $n$ is the power-law constant. For uniaxial deformation, $\dot{\varepsilon}$ and $\sigma$ refer to that deformation; more generally, they are the second invariants of the strain-rate and deviatoric-stress tensors. A weak dependence on pressure often is ignored, or is allowed for by referring $T$ to the in situ melting point (Weertman, 1968; Doake and Wolff, 1985).

For dislocation glide, $n$ should be equal to 3 based on the following analysis, summarized from the review by Weertman (1973). The strain rate is simply the product of the rate at which dislocations move through a material, their abundance and the deformation achieved per dislocation, or

$$
\dot{\varepsilon}=\alpha \rho b \nu
$$

where $\alpha$ is a geometric factor of the order of $1, \rho$ is the dislocation density, $b$ is the Burgers vector of the dislocations (approximately the molecular spacing) and $\nu$ is the dislocation velocity. The dislocation velocity is, both theoretically and observationally,

$$
\nu=\gamma \sigma \exp \left[-\frac{Q}{k T}\right]
$$

where $\gamma$ is a constant and $\sigma$ is the applied stress causing dislocation glide. The dislocation density, $\rho$, for steadystate deformation is approximately that which gives an internal stress from the dislocations equal to the applied stress. The internal stress is $\mu b /(\beta d)$, where $\beta$ is a constant of the order of $1, \mu$ is the shear modulus, and $d$ is the dislocation spacing and is related to the dislocation density by $\rho \approx 1 / d^{2}$. Then

$$
\rho \approx\left[\frac{\beta \sigma}{\mu b}\right]^{2} .
$$

Combining Equations (2)-(4) yields

$$
\dot{\varepsilon}=\frac{\alpha \beta^{2} \gamma}{\mu^{2} b} \exp \left[-\frac{Q}{k T}\right] \sigma^{3}
$$

which is essentially Equation (1) with a more physical statement of $A_{\circ}$ and with $n=3$.

This result has broad support from laboratory and field observations, especially at high stresses $(\sigma>\approx 1$ bar $)$ (Weertman, 1973; Hobbs, 1974; Budd and Jacka, 1989); results at lower stresses $(\sigma<\approx 1$ bar $)$ are equivocal as to whether $n=3$ or $n<3$ (including $n=1$ ), probably because of the great difficulty in conducting such tests (see Weertman, 1973; Budd and Jacka, 1989). 
C. If temperature, stress and cumulative strain are small enough but dislocation glide still dominates, the strain rate increases approximately with the first power of the stress $\{2\}$

This is the regime of Harper-Dorn creep. From the equations above, it is evident that, if dislocation density is constant, then linear-viscous behavior is predicted. Constant (stress-independent) dislocation density may apply at very small strains before dislocation multiplication has begun, or at very small stresses and strain rates, which may not be large enough to cause dislocation multiplication above the initial annealed concentration (see Energetics, below) or which may allow recovery (principally diffusional) processes to "heal" dislocations as fast as they are produced.

There is little doubt that creep exists with $n=1$. There is greater uncertainty whether $n=1$ creep is steady, and whether it occurs in ice under natural conditions (see Weertman, 1985). A number of workers have reported $n=1$ for natural ice (or at least $n<3$ ) (see references in Weertman, 1973; also Doake and Wolff, 1985; Pimienta and Duval, 1987), but this point is by no means settled (Paterson, 1985; Budd and Jacka, 1989).

\section{RESULTS - GRAIN GROWTH}

A. The average grain-size in an existing population of grains always increases (grain growth always occurs), although creation of new, smaller grains may cause the average grain-size to decrease or remain constant over time $\{4\}$

Grain growth in ice is driven primarily by two factors: the curvature of energetic grain boundaries, and differences in stored strain energy between grains. The curvature of grain boundaries that possess a surface tension causes what amounts to a pressure difference between adjacent grains, with the higher pressure on the concave side of the boundary (usually the smaller-grain side). Molecules tend to jump from the high-pressure side to the low-pressure side, causing the boundary to move toward the highpressure side. The process usually accelerates with time; as the boundary moves toward the center of a small grain, that grain becomes smaller, the curvature of its boundary increases, and the migration speeds up until the smaller grain is consumed entirely. The average grain-size in the sample is increased every time one grain consumes another in this fashion.

Boundary migration can be stopped or slowed by second-phase particles, pores or dissolved impurities at high concentrations; however, concentrations found in natural glacier ice rarely or never are high enough to stop grain growth (see review by Alley and others, 1986a, b). Grain growth also can be slowed significantly if boundaries assume special orientations that cause them to have low energy (see Grain Growth B, below), but this also does not stop grain growth. The tendency for boundary migration from curvature and tension exists and will always tend to cause grain growth until arrested by second-phase particles and other factors, which in typical ice-sheet ice would require average grains about
$1 \mathrm{~m}$ across or larger (Alley and others, 1986b). If grain growth in a polycrystal with ordinary, high-angle grain boundaries is driven by the curvature and surface tension of boundaries and if the effects of contaminant materials are not large, then the cross-sectional area of grains increases linearly with time; this is the regime of normal grain growth (e.g. Gow, 1969; Alley and others, 1986a).

Boundary migration also can be caused by differences in stored strain energy across grain boundaries. Dislocations and other defects introduced into a crystal lattice by strain increase its energy. Because boundary migration reconstitutes the ice through which the boundary moves, the ice just behind a moving boundary has not been deformed and thus is unstrained and has low energy. (For very rapid migration, excess vacancies may enter the lattice behind a moving grain boundary, raising its energy and slowing the boundary (Gleiter, 1979), but this is unlikely to be a significant factor under typical conditions in ice sheets (Alley and others, 1986a).) When a boundary separates strained from unstrained ice, the free energy of the strained side is higher, and the total energy can be lowered if the boundary migrates into the strained grain. Once started, the migration of a boundary in deforming ice will tend to continue because the ice immediately behind it is unstrained and that in front is older and thus more strained. Eventually, a strained grain being consumed in this fashion will become small enough that curvature effects also are important, and it will be consumed rapidly. (Initially, sufficiently large differences in stored strain energy can cause a boundary to move away from its center of curvature, away from a smalll unstrained grain into a large strained grain, in the opposite direction of that expected from curvature alone. As the strained grain becomes small, both strain energy and curvature work in the same direction, causing the strained grain to be consumed. Consumption of any grain always increases the average grain-size.)

It thus appears that if grain boundaries are not pinned by second-phase particles, they always migrate. And, if new grains are not created, migration of boundaries inevitably leads to grain growth. The only ways to avoid this are if each grain consumes a neighbor at the exact same rate that another neighbor consumes it, or if all grains have the same strain energy and are perfectly the same size and shape and perfectly packed, so that all boundaries are exactly straight. Real materials always are imperfect and have some randomness, so grain growth always occurs.

B. If the average grain-size is not increasing (or is not increasing as rapidly as expected for normal grain growth), then existing grains are being subdivided (polygonization) or entirely new grains are being created (recrystallization) $\{4\}$

Consumption of some grains by others without increase in the average grain-size in a constant-density material requires that new grains are formed to replace those lost. Two mechanisms are believed to dominate such newgrain formation: polygonization and recrystallization ("rotation recrystallization" and "migration recrystallization", respectively, in the terminology of Poirier (1985, chapter 6)). 
Polygonization is the formation of new grains by subdividing the old grains. If different parts of a grain are subjected to different stress states (virtually guaranteed by grain-grain interactions in a polycrystal), then the grain can become bent or twisted. Dislocations tend to organize between relatively undeformed regions called sub-grains to form sub-grain boundaries that relieve this bending or twisting and lower the energy of the system. If a sub-grain boundary becomes sufficiently strong (or a sub-grain becomes sufficiently rotated), then the boundary becomes a full grain boundary; the division between sub-grains and grains is somewhat arbitrary. Low-angle grain boundaries produced by polygonization initially cause a small mismatch between grain lattices, which means that the boundaries have low energy and migrate slowly under curvature-induced stress. Continued deformation can increase the angle of mismatch. Polygonization often can be identified by occurrence of more nearest-neighbor grains with small misorientations than would occur randomly in a sample. (Poirier (1985) distinguished between the formation of new grains by subdividing, which he called "creep polygonization", and the refinement of this structure by further deformation, which he called "rotation recrystallization". Here I use "polygonization" to refer to both.)

Recrystallization ("migration recrystallization" of Poirier (1985)) is the production of new grains at initially high angles to their neighbors. The new grains typically nucleate from existing grain or sub-grain boundaries separating regions with different stored strain energy, with the boundary bowing out toward the more strained side. The stored strain energy in the region ahead of the migrating boundary must be large enough to overcome the increase in grain-boundary energy caused by this bowing out of the boundary (see Energetics, below). The new grains in recrystallization usually form in energetically favored orientations with their $c$-axes at high angles $\left(\approx 45^{\circ}\right)$ to compressional axes (e.g. Kamb, 1972).

\section{Growth selection does not form fabrics over broad ranges of normal grain growth $\{3\}$}

The energy of grain boundaries is nearly independent of relative grain orientations, except for certain favored orientations (e.g. twin boundaries, small misorientations) which may reduce the boundary energy by more than an order of magnitude (see review in Verhoeven, 1975, chapter 7). This opens the possibility that, if two grains with similar orientations grow together, one will not consume the other rapidly because of the low boundary energy. Repetition of such growing together of similarly oriented grains could yield regions in which grains exhibit a preferred orientation. However, simple calculations show that the favored orientations are sufficiently rare that this is unlikely to matter in natural ice, and indeed, no strong fabrics are observed during normal grain growth.

Growth selection may contribute to fabrics when stored strain energy is higher than in normal grain growth. For example, polygonization produces low-angle boundaries, and the grains across such boundaries are consumed only slowly. However, the new grains will occur within an area that started as a single grain, and boundaries between such areas are high-angle grain boundaries that can grow. In this way, relatively large areas of grains may develop with similar orientations. Also, it is possible that grains in some orientations will store more strain energy than those in other orientations. The high-energy grains would be consumed in grain growth, leaving grains with a preferred orientation. In the first case, any fabric measurement that averaged over a large enough area would be unaffected by growth selection. The second case might matter, but is essentially unexplored in ice.

\section{RESULTS — FABRIC FORMATION}

\section{A. During ice deformation, $c$-axes always rotate toward compressional axes and away from ten- sional axes $\{4\}$}

Deformation parallel to the basal plane in ice is $100-1000$ times easier than in any other direction (Weertman, 1973). An applied stress thus causes basal glide for almost any orientation, which tends to cause grain deformation. However, in a polycrystal, the probability that adjacent grains will deform in exactly the same direction is vanishingly small (except in the limit of large strains in simple shear) (Fig. 1). Even if the deformation is in the same direction in adjacent grains, the active slip planes are unlikely to match exactly across the grain boundary. Adjacent grains then interfere with the deformation of any given grain. This interference can be avoided if the grain rotates at the same time that it deforms in basal glide. The adjustment between two grains is taken up by slip along the grain boundary, which allows sliding easily (Ignat and Frost, 1987). The result is grain deformation on the basal plane, grain elongation, and rotation of the normal to the glide plane (the $c$-axis in ice) toward compressional and away from tensional axes (Figs 1 and 2). This result is general for essentially any crystal deforming by dislocation glide and constrained by a testing apparatus or adjacent grains in a polycrystal (e.g. Verhoeven, 1975, chapter 3).

\section{B. Rotation of existing grains in polycrystals is described to good approximation by the results of Azuma and Higashi (1985) $\{2\}$}

Azuma and Higashi (1985) deformed thin ice specimens uniaxially and found that the strain in each grain was approximately proportional to the resolved shear stress along its basal plane, with the bulk strain equal to the strain of individual grains averaged over the entire sample. They then found that this model predicts the observed fabric development at Dye 3, Greenland, in the region of normal grain growth, where such a model for an existing population of grains should apply. More recently, Lipenkov and others (1989) have found that the Azuma and Higashi (1985) model, modified for uniaxial tension following Fujita and others (1987), accurately describes fabric observations in the Vostok, East Antarctica, core.

Briefly, under uniaxial tension or compression, the resolved shear stress on the basal plane of an ice crystal is 

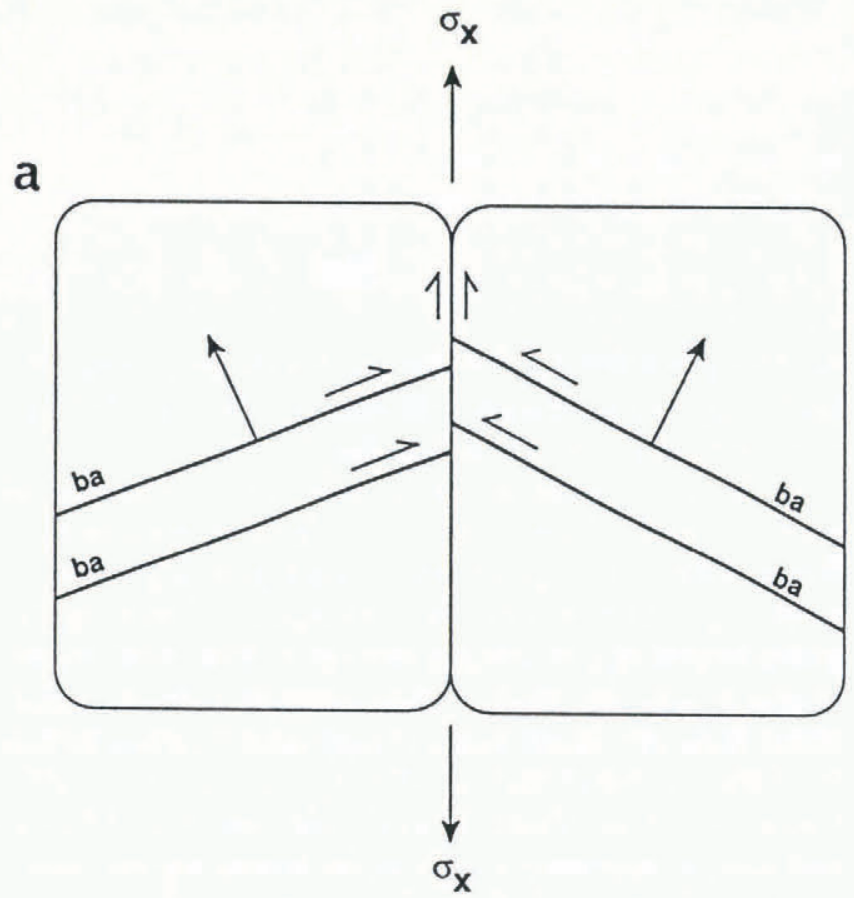

b

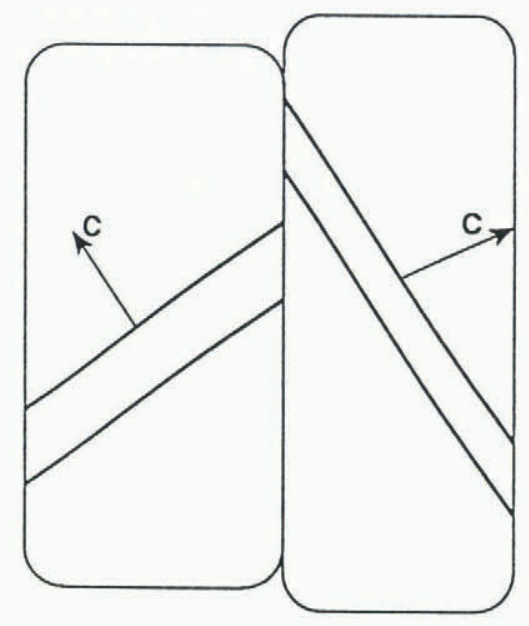

Fig. 1. Cartoon showing grain rotation from deformation of a polycrystal. In (a), uniaxial tension $\sigma_{x}$ causes resolved shear stresses on the basal planes, $b a$, of two grains, which cause mutual interference for basal glide. Deformation is achieved by basal glide plus rotation of caxes, $c$, away from the tensional axis, leading to the situation in (b).

proportional to the geometric factor $S=\cos \phi_{0} \sin \phi_{0}$, where $\phi_{0}$ is the angle between the $c$-axis of a grain and the unique stress axis. A grain that elongates from length $l_{0}$ to length $l$ has incremental strain $\Delta \epsilon_{\mathrm{g}}=\left(l-l_{\mathrm{o}}\right) / l_{\mathrm{o}}$ and its $c$ axis rotates from angle $\phi_{\mathrm{o}}$ to angle $\phi$ according to $\cos \phi=\cos \phi_{\mathrm{o}} l_{\mathrm{o}} / l$ for uniaxial tension and $\sin \phi=$ $\sin \phi_{0} l / l_{0}$ for uniaxial compression. An incremental strain $\Delta \epsilon$ averaged over the entire sample causes strain in an individual grain $\Delta \epsilon_{\mathrm{g}}=\Delta \epsilon S / \bar{S}$ where $\bar{S}$ is the average of the geometric factor $S$ over all grains in the sample. Combining these relations yields

$$
\cos \phi=\frac{\cos \phi_{\mathrm{o}}}{1+(\Delta \epsilon S / \bar{S})}
$$

for rotation of a grain in uniaxial tension, and

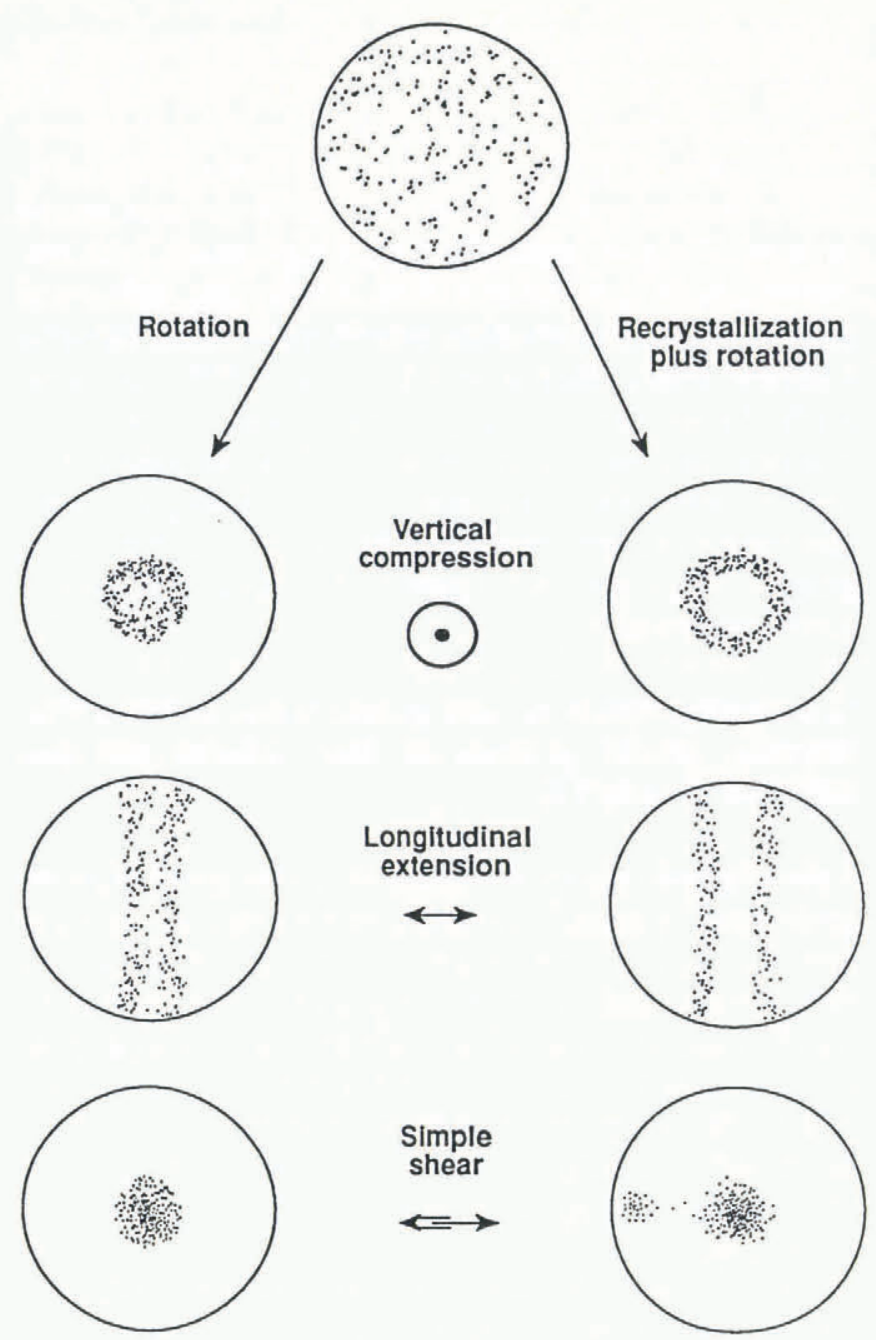

Fig. 2. Schematic evolution of c-axis fabrics as a function of stress state and active processes. An initial random distribution (top) yields solid-cone distributions (left) if rotation alone is active, and girdle/surface-cone distributions (right) if recrystallization also is active.

$$
\sin \phi=\sin \phi_{\mathrm{o}}\left(1+\Delta \epsilon \frac{S}{\bar{S}}\right)
$$

for rotation in uniaxial compression; pure shear is the superposition of these, and simple shear is pure shear plus a rigid-body rotation. The parameter $\bar{S}$ is the geometric softness of an ice sample to deformation, arising from the average resolved shear stress on basal planes. Numerical integration of Equations (6a) or (6b) for uniaxial deformation shows that $\bar{S}$ generally decreases with increasing strain, but that strains in excess of $100 \%$ are needed to halve $\bar{S}$ (Alley, 1988); we will find later that uniaxial deformation without recrystallization usually does not persist to strains significantly greater than $100 \%$, so that the fabric effects from uniaxial deformation are not large, but that simple shear does reach larger strains and affect ice hardness significantly through fabric development.

The main principle underlying Equations (6) is that the strain in any grain is proportional to the resolved shear stress on its basal plane; the rest of the derivation is simply geometry. This is not a necessary result; in some materials at some temperatures, the strain in individual 
grains may be nearly independent of orientation, or the strain may be such as to minimize differences in deformation between adjacent grains, or some intermediate behavior (e.g. Etchecopar, 1977; Lister and others, 1978). Strain proportional to resolved shear stress would be most likely at relatively high temperatures and low stresses, as in ice. Under these conditions, diffusional processes can accommodate differences in strain between adjacent grains (Duval and Lliboutry, 1985), allowing grain elongations to be nearly independent of neighbors, although still with constraint from neighbors sufficient to force rotation. This adds some theoretical support to the laboratory and field results that Equations (6) do describe grain rotation in ice. However, further data on this point would be desirable.

\section{In polygonization, new grains form within a few degrees of old grains, so that fabrics are not affected greatly $\{4\}$}

This is almost true by definition. If a new grain forms by progressive sub-grain rotation, and is defined to have formed when it has reached an angle of a few degrees, then the new grain will plot very close to the grain from which it formed on a $c$-axis fabric diagram, causing little change in the overall fabric. Polygonization does not remove the old grains, and the boundary between a new grain and its parent may have low enough energy to slow consumption of one by the other.

\section{New grains in recrystallization form with $c$ - axes midway between compressive and tensile axes, causing formation of new grains to change fabrics $\{3\}$}

During recrystallization, new grains are believed to form in energetically favorable postions with $c$-axes approximately bisecting the angle between compressional and tensional axes, so that resolved shear stress on basal planes is maximized (see Kamb, 1972). This produces a girdle about the unique axis in compression and tension, and two concentrations in the plane containing the compressional and tensional axes in pure shear and simple shear (and usually with greater scatter of positions perpendicular to this plane than parallel to it. For simple shear, this means that one of the concentrations is normal to the shear plane, and the other is almost, although generally not exactly, in the shear plane) (Fig. 2).

The new grains are relatively strain-free and consume older grains by boundary migration. The new grains also accumulate strain energy while rotating and deforming, and eventually are consumed by newer grains. Recrystallization thus tends to replace an existing population of grains with a new population of grains which have $c$-axes that on average are rotated a little toward compressional axes from nucleation positions midway between tensional and compressional axes.

\section{ENERGETICS}

The energies involved in normal grain growth, steadystate creep and nucleation of new grains provide considerable insight into how these processes proceed. I discuss these next.

The driving stress for migration of a typical, spherically curved grain boundary of radius of curvature $r$ separating grains with equal strain energies is

$$
E_{\mathrm{gb}}=\frac{2 \gamma_{\mathrm{gb}}}{r}
$$

where $\gamma_{\mathrm{gb}}=0.065 \mathrm{~J} \mathrm{~m}^{-3}$ is the grain-boundary energy for high-angle boundaries (Hobbs, 1974, p. 440). If $r=1 \mathrm{~mm}$, this amounts to $130 \mathrm{~J} \mathrm{~m}^{-3}$.

A boundary will migrate away from its center of curvature only if some other driving stress exceeds this. Recrystallization requires that small grains grow into large grains, and thus that the stored strain energy in old grains exceed $E_{\mathrm{gb}}$, or around $100 \mathrm{~J} \mathrm{~m}^{-3}$. And, if the nuclei of new grains are smaller than assumed here, as seems likely, then the stored strain energy must be even higher to drive recrystallization. (Duval and others (1983) conducted this analysis and assumed $r=0.1 \mathrm{~mm}$, obtaining a necessary stored strain energy for recrystallization of near $1000 \mathrm{~J} \mathrm{~m}^{-3}$. Typical grain-sizes in recrystallization are near $2 \mathrm{~mm}$ (although with some variation as a function of temperature and deviatoric stress; Jacka, 1984a), so the nuclei cannot be much bigger than $1 \mathrm{~mm}$ and easily could be smaller.)

In steady-state creep, a density of dislocations, $\rho$, contributing to deformation is expected that gives an internal stress from the elastic fields of the dislocations equal to the magnitude of the applied stress, as given in Equation (4) (Weertman, 1973). The stored energy associated with this density, $E_{\mathrm{d}}$, is for screw dislocations (Weertman and Weertman, 1964, p. 46-47)

$$
E_{\mathrm{d}}=\rho\left(\frac{\mu b^{2}}{4 \pi} \ln \left(\frac{R}{5 b}\right)+e_{\mathrm{c}}\right)
$$

where $\mu=3 \times 10^{9} \mathrm{~Pa}$ is the shear modulus, $b=$ $4.5 \times 10^{-10} \mathrm{~m}$ is the Burgers vector, $R$ is the radius over which the strain energy acts, and a dislocation core of radius $5 b$ has energy $e_{\mathrm{c}}$ which typically is $10-20 \%$ of the elastic strain energy represented by the first term in Equation (8). Poirier (1985, p. 52) suggested using $R=1 / \rho^{0.5}$; Weertman and Weertman (1964, p. 47) favored either the grain-size or the sub-grain-size for $R$, yielding a larger energy. However, because $R$ appears in a logarithmic term, this difference is not critical. For an applied stress of $\sigma=1 \mathrm{bar}$, a grain-size of $1 \mathrm{~mm}$, a subgrain-size assumed to lie between the grain-size and the mean dislocation spacing, and the core energy $e_{\mathrm{c}}$ taken as $10-20 \%$ of the elastic energy, Equations (4) and (8) yield a steady-state dislocation strain energy of between 2 and $4 \mathrm{~J} \mathrm{~m}^{-3}$. This would be increased by $50 \%$ for edge dislocations (Weertman and Weertman, 1964, p. 50). In general, then, the steady-state strain energy at 1 bar is less than that needed to drive recrystallization by more than an order of magnitude, and possibly by two orders of magnitude. A stress of 2 bar would allow average strain energy to drive recrystallization only if the calculations here err in the proper directions by an order of magnitude, and a stress of about 7 bar would be needed to drive recrystallization if the calculations here are correct. However, Jacka (1984b) found good evidence for 
recrystallization at stresses as low as $1.3 \mathrm{bar}$ at $-18^{\circ} \mathrm{C}$. Clearly, there is a possible problem here.

At least four mechanisms may contribute to solving this problem. First, the factor $\beta$ in Equation (4) is not known accurately, and enters the calculation of stored strain energy as a squared term, so relatively large errors in these calculations are possible. For example, data shown by Weertman (1968, fig. 12) for iron suggest that $\beta=2$ is more accurate than $\beta=1$, although for an ironsilicon alloy $\beta=1$ appears more accurate.

Secondly, Duval and others (1983) pointed out that stored energy is likely to be higher in some grains than in others, and nucleation of strain-free grains would occur adjacent to the most strained grains. Thus, recrystallization requires not that the average grain have large stored strain energy, but that the extreme grains do.

Thirdly, the steady-state dislocation density of Equation (4) refers to dislocations of one sign contributing to creep deformation. If dislocations of opposite signs accumulate in tangles or sub-grain boundaries, then they contribute to the stored energy field for grain growth but not to the long-range internal elastic stress field balancing the applied stress, and thus not to the steady-state density of Equation (4). Stored energy then can increase above that calculated for steady-state creep. For example, Equation (4) with a stress of $10^{5} \mathrm{~Pa}(1 \mathrm{bar})$ and $\beta=1$ yields a dislocation density of $5 \times 10^{9} \mathrm{~m}^{-2}$, and with a stress of $10^{4} \mathrm{~Pa}$ yields a density of only $5 \times 10^{7} \mathrm{~m}^{-2}$. Annealed ice with especially low dislocation densities may have around $10^{8} \mathrm{~m}^{-2}$ (Hobbs, 1974, p. 250-51), but lower values are difficult to achieve and typical annealed materials usually have dislocation densities in the range $10^{9}-10^{10} \mathrm{~m}^{-2}$ (Dieter, 1976, p. 144). Thus, except at high stresses, the typical dislocation density may exceed the value calculated from Equation (4) needed to balance the applied stress, increasing the stored strain energy above that calculated from Equations (4) and (8). (If the elastic stress fields from the residual dislocations in annealed ice exceed the applied stress, as seems possible in ice at low stresses, then they may slow or stop dislocation multiplication. This would lead to the constant dislocation density needed for $n=1$ creep, or to a weaker dependence of density on stress than in Equation (4) and thus $n<3$ creep.)

Finally, it remains possible that the recrystallization nuclei form in twin orientations to the grains into which they grow (Matsuda and Wakahama, 1978). This could reduce the grain-boundary energy, $\gamma_{\mathrm{gb}}$, by more than an order of magnitude (Verhoeven, 1975, p. 188), thus allowing recrystallization at a lower driving stress. Good, quantitative theories are not available for any of these mechanisms in natural ice, but it seems likely that some combination of these mechanisms can explain the apparent contradiction of recrystallization observed at stresses that are not calculated to be large enough to store enough strain energy to cause recrystallization.

Before finishing this section on energetics, it is worth noting that the stored strain energy needed for polygonization is not constrained well. Polygonization requires production of new (but low-angle and low-energy) grain boundaries, and thus the introduction of their grainboundary energy. However, this provides no information on how strain energies vary in sub-grains between sub- grain boundaries, where Equation (4) is presumed to apply.

\section{SYNTHESES}

The discussions above focus on individual phenomena (ice creep, grain growth, fabric development), but all are occurring at the same time within a material. Here I briefly re-organize the discussion above to show the interrelationships of these processes. These interrelationships are shown graphically in Figure 3.

A. If normal grain growth is occurring (that is, if the cross-sectional area of grains is increasing linearly with time, the grain-size distribution is unimodal and grains are equant and convex, with no strain shadows at least initially) then:

No new grains are being added to the population.

Stored strain energy is small compared to grainboundary energy.

$c$-axis fabric development occurs by grain rotation, probably following the Azuma and Higashi (1985) hypothesis as extended by Alley (1988).

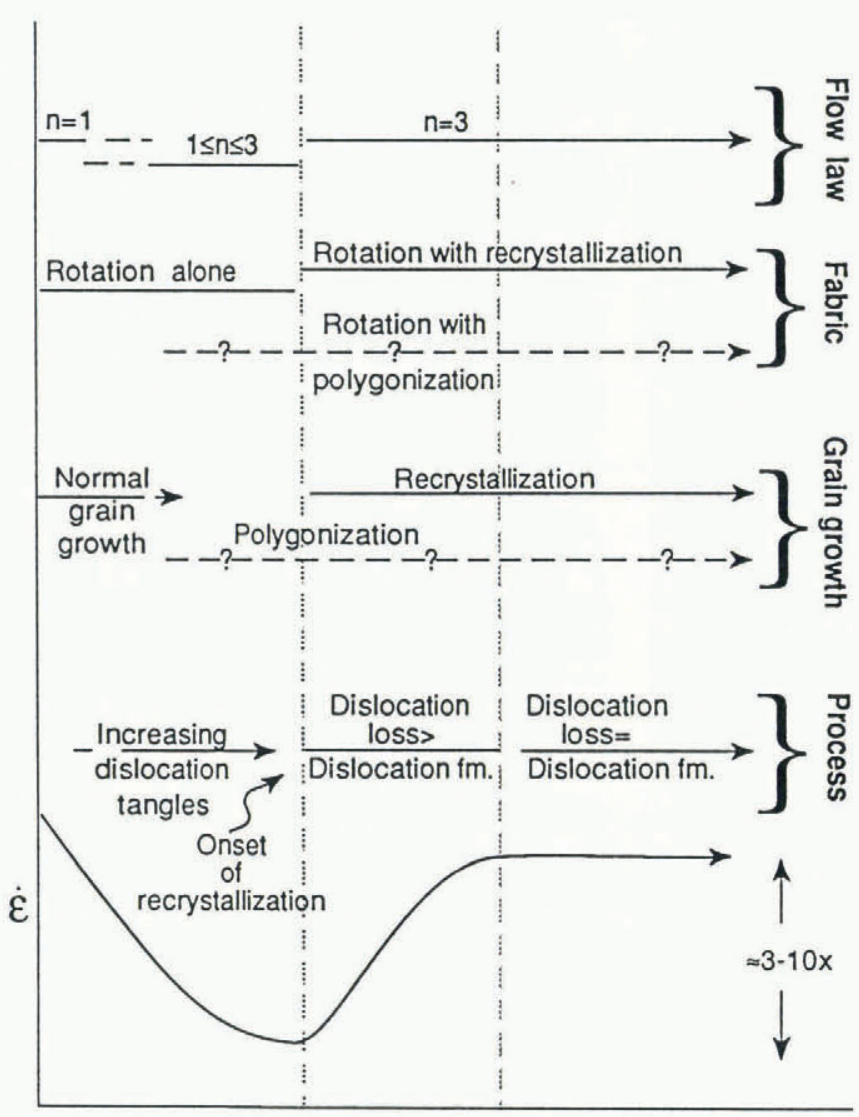

$\varepsilon$

Fig. 3. Schematic curve of strain rate versus cumulative strain for constant-stress deformation of polycrystalline ice, following Budd and Jacka (1989). Flow-law exponents, fabric controls, grain-growth behavior and microscopic processes are indicated. 
The density of dislocations causing shear on the basal plane is not known in comparison to the steady-state density of Equation (4); hence, the power-law exponent, $n$, is not known and could be 1,3 or some intermediate value.

If a nearly constant strain rate is maintained in the field or the laboratory without rigid-body rotation (e.g. if uniaxial deformation or pure shear is maintained but not simple shear), this regime eventually must break down. Grain rotation progressively aligns grains toward a position in which they have no resolved shear stress on their basal planes, requiring increasing stress to maintain the strain rate and eventually causing recrystallization. However, strain of about $100 \%$ is needed to cause a doubling of the ice hardness (Alley, 1988).

\section{B. If $c$-axis fabrics are tightening, strain shadows and sub-grains appear, and grain growth is slower than normal (including zero or negative growth), then:}

Either (i) polygonization is occurring, allowing fabric development to continue to follow the Azuma and Higashi (1985) hypothesis closely; or (ii) recrystallization is occurring, but is sufficiently slow compared to grain rotation that the rotation dominates the fabric development.

And, strain energy has not reached the level of D, below, but may be less than, equal to or greater than the value of Equation (4), so that $n$ is not constrained well.

This regime must change as the fabric tightens in uniaxial deformation or pure shear if strain is to continue.

\section{If a strong single-maximum fabric develops in simple shear, together with slow, zero or negative grain growth and with strain shadows or sub- grains, then:}

Either (i) polygonization is occurring, or (ii) recrystallization is occurring, but is sufficiently slow compared to grain rotation that the rotation dominates the fabric development.

And, the level of stored strain energy relative to Equation (4) is unknown, as is the value of $n$.

A steady state of deformation is possible.

\section{If a girdle fabric develops during active deformation, together with zero grain growth and with strain shadows, then:}

Recrystallization is occurring.

Stored strain energy is steady and large.
Active dislocations almost certainly satisfy Equation (4), giving $n=3$ in power-law creep.

A steady-state of deformation is possible.

\section{ICE-SHEET OBSERVATIONS}

The different regimes described above occur in natural ice sheets and have been delineated based on this occurrence. It next is useful to describe their occurrence. Data are available from a number of locations, including (but not limited to) Byrd, Little America (Gow and Williamson, 1976), Vostok (Pimienta and Duval, 1987), Mizuho (Fujita and others, 1987) and Law Dome (Russell-Head and Budd, 1979) in Antarctica; Camp Century (Herron and Langway, 1982) and Dye 3 (Herron and others, 1985) in Greenland; and the Barnes Ice Cap in Arctic Canada (Hooke and Hudleston, 1980). However, considering the broad range of glacial environments with differing temperatures, stress states and histories, this is a very small sample. Recent reviews and syntheses include Hooke and Hudleston (1980), Lliboutry and Duval (1985) and Budd and Jacka (1989).

As a general rule, the upper layers (typically 100$1000 \mathrm{~m}$ thick) of the central regions of large ice sheets exhibit normal grain growth. Below this is a region where grains do not grow but girdle fabrics do not form. This is followed downward by a single-maximum, simple-shear region with small grain-size, which often (although not always) corresponds to Wisconsinan/Weichselian ice. If the basal ice is warm, this may be replaced downward by a region of very large grains with a multiple-maximum girdle fabric, which may be caused by annealing associated with fluctuating stresses (Budd and Jacka, 1989). Ice shelves below the firn are characterized by recrystallization girdle fabrics (Fig. 4).

Attempts to quantify the strain rates or stresses, cumulative strains, temperatures, etc. that delineate the boundaries of some or all of these different regimes have been presented, e.g. by Hooke and Hudleston (1980), Alley (1988) and Budd and Jacka (1989). These may

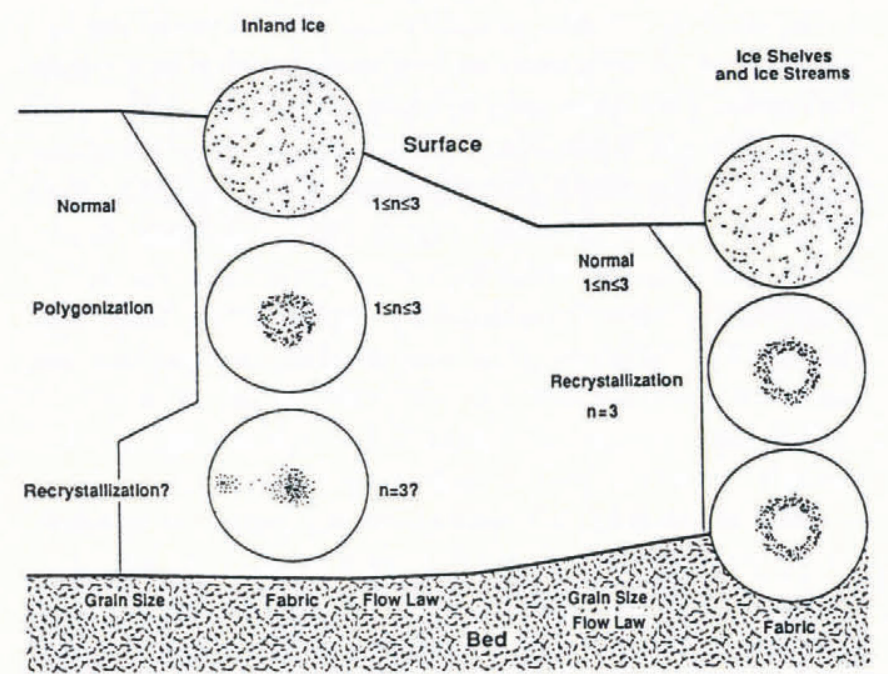

Fig. 4 Simplified model for grain growth, fabric development and flow-law exponent in a cold ice sheet. 
provide useful guidelines, but the inadequacy of the data base and the complex interrelationships of the control parameters must be remembered at all times.

The symmetries of $c$-axis fabrics observed in ice sheets can be described reasonably well with a simple model (Figs 2 and 4): firn has nearly random fabrics, deformation under normal stresses causes rotation toward compressional axes and away from tensional axes, deformation plus rotation in simple shear causes rotation toward the normal to the shear plane, polygonization causes fabrics to evolve in a similar way to rotation alone, and recrystallization causes grains to nucleate with $c$-axes between compressional and tensional axes and then to rotate as above, with old grains consumed before they have rotated through large angles. Under normal stresses, existence of $c$-axes near compressive axes shows that recrystallization is inactive or slow; a girdle about compressive axes shows that recrystallization is fast. Under normal stresses without recrystallization, compression in one direction with equal tension in the other two directions (uniaxial compression) causes $c$-axis rotation toward the compression axis, tension in one direction with equal compression in the other two directions (uniaxial tension) causes $c$-axis rotation toward the compression plane, and tension in one direction, compression normal to it, and neutral stresses in the third direction (pure shear) causes $c$-axis rotation toward the compressional axis with rotation fastest for $c$-axes in the plane of the compressional and tensional axes. Recrystallization causes girdles, around the compressional axis in uniaxial compression and the compressional plane in uniaxial tension, with a modified girdle about the compressional axis in pure shear, such that the girdle is elongated in the direction of the neutral axis but has few or no $c$-axes near the plane containing the compressional and neutral axes. Simple shear without recrystallization causes $c$-axis rotation toward the normal to the shear plane, and recrystallization in simple shear produces $c$-axes normal to the shear plane and near the shear plane nearly parallel to the shear direction. Excellent diagrams have been presented by Budd and Jacka (1989) for many of these; also see Gow and Williamson (1976), Alley (1988) and Figures 2 and 4 in this paper.

\section{HYPOTHESES}

The goal of this long tour through the texture, fabric and deformation of ice was to constrain possible flow laws for natural ice deforming in regimes not readily accessible to laboratory measurement. My central thesis is that the macroscopic processes of grain growth and fabric formation are easier to observe than the microscopic processes (dislocation glide, diffusion, etc.) that control them, that these microscopic processes also control deformation behavior, and that we should be able to use the observed characteristics of ice cores to infer microscopic processes and, from them, the deformation behavior and flow law.

We find a broadly consistent picture, but one which leads to a clear indication of the flow law only in certain special cases. For most glacial ice, my theoretical understanding of the microscopic processes is not sufficiently advanced to allow the rather crude macroscopic indicators to distinguish between models. Here, I summarize my understanding of the macroscopic and microscopic regimes in broadly (and nebulously) defined regions, and I suggest hypotheses to test for the flow law.

\section{Normal grain-growth region - hypothesis: $n=1$, but $1 \leqslant n \leqslant 3$ possible. Fabric enhancement from Alley (1988)}

In the upper 100-1000 $\mathrm{m}$ of the central regions of cold ice sheets, stored strain energy is small compared to grainboundary energy. Deformation occurs by dislocation glide on basal planes and probably is accommodated by diffusional processes. $c$-axes of grains rotate toward compressional axes during deformation. No new grains are produced. The $c$-axis fabric then records the cumulative strain (by its tightness about the compressional axis) and the stress state (by its shape and orientation), and affects the ice hardness (through the fabric effect on the prefactor $A_{\circ}$ in Equation (1)), probably as sketched by Alley (1988).

The best estimate of the dislocation density needed for steady-state creep would not produce enough strain energy to affect normal grain growth; hence, we lack a direct probe to distinguish between the steady-state dislocation density (which gives $n=3$ in Equation (1)) and the case where diffusional recovery processes or other processes balance or prevent dislocation multiplication and yield $n=1$. It is noteworthy, however, that recovery processes must be active or dislocation multiplication must be suppressed, because the normal grain-growth regime can persist to $\approx 10-100 \%$ strain or possibly more (Gow and Williamson, 1976; Herron and others, 1985; Lipenkov and others, 1989), whereas at higher stresses or temperatures in the laboratory $\approx 1 \%$ strain initiates recrystallization, reaching a steady state of deformation by $\approx 10 \%$ strain (Jacka, 1984b). Recovery processes remove dislocations and reduce dislocation density. This, combined with reasonably good evidence for $n=1$ at low stresses in laboratory experiments on other materials (see Weertman, 1985) and a suggestion of $n=1$ from laboratory and field observations on ice (Weertman, 1973; Doake and Wolff, 1985; Pimienta and Duval, 1987), make $n=1$ the leading candidate for the flow law during normal grain growth. However, $1 \leqslant n \leqslant 3$ is possible, including the possibility that $n$ lacks a physical meaning, but only is a curve-fitting parameter for creep controlled by a changing balance of processes.

\section{Polygonization region - hypothesis: $n=1$, but $1 \leqslant n \leqslant 3$ possible. Fabric enhancement from Alley (1988)}

Deeper than the normal grain-growth regime, but still in approximately the upper one-half to two-thirds of the central regions of large ice sheets, grain-size from at least some sites (Gow and Williamson, 1976; Herron and others, 1985) is nearly independent of depth and age, abundant sub-grains and strain shadows occur, but fabrics are similar to and slightly tighter than those in the normal grain-growth region. This behavior probably 
arises from polygonization. That is, dislocations organize into sub-grain boundaries to reduce their energy, and with increasing deformation these sub-grain boundaries strengthen until they cross an arbitrary threshold to become grain boundaries. The new grains have similar orientations to old grains, so fabrics can continue to tighten with deformation. Lack of girdle fabrics shows that old grains are not being consumed by new, highangle nuclei. The easiest explanation is that recrystallization is slow or absent. Cumulative strains can approach or slightly exceed $100 \%$ in this region without causing rapid recrystallization, whereas $1-10 \%$ strain causes recrystallization during more rapid deformation in the laboratory (Jacka, 1984b; Jacka and Gao, 1989), showing that recovery processes continue to be important; however, the sub-grains and polygonization attest to increasing stored strain energy compared to normal grain growth. Because recovery processes are active, $n=1$ remains possible, although the increasing stored strain energy makes $n=3$ more likely than for normal grain growth.

Laboratory experiments (e.g. Jacka, 1984b) show that uniaxial deformation causes ice to harden by a factor of roughly $2-10$ with increasing strain up to about $1 \%$, prior to initiation of recrystallization, with this hardening probably caused by development of dislocation tangles (Fig. 3). If $n=1$, then such tangles should not be significant in the interiors of sub-grains, and the ice hardness should be unchanged from the normal graingrowth regime, except for fabric changes that can be treated in the same way as in that regime. If $n>1$, then it is likely that dislocations are building up within the subgrains, and they may become tangled and harden the ice. Then it is possible that $A_{\mathrm{o}}$ should be decreased somewhat independent of fabric.

\section{Simple-shear region - hypothesis: $n=3$, but $1 \leqslant n \leqslant 3$ possible. Fabric enhancement from Budd and Jacka (1989, fig. 9)}

Still deeper, the basal shear stress comes to dominate over the normal stresses, so simple shear occurs. The fabric is dominated by a strong vertical single maximum, with grain-size small and relatively constant. A critical question is whether a second, weaker maximum exists.

As discussed above, laboratory experiments (e.g. $\mathrm{Kamb}, 1972$ ) show that recrystallization in simple shear produces nuclei in two positions: one strong maximum with $c$-axes normal to the shear plane, and one weaker maximum with $c$-axes nearly parallel to the shear direction and nearly parallel to the shear plane. $c$-axes in the stronger maximum will tend to stay there and caxes in the weaker maximum will tend to rotate toward the stronger one.

If many nuclei are being formed near the horizontal position, they should be observable as a second maximum, even if weak and spread out through rotation. However, if polygonization is occurring without recrystallization, then only one maximum should exist. As discussed below, occurrence of two maxima argues for $n=3$, whereas one maximum leaves open the question of $n$ between 1 and 3 , inclusive.

Available data are equivocal. Some observations show no clear evidence of a second maximum (e.g. $1302 \mathrm{~m}$ in the Byrd core; Gow and Williamson, 1976), but others do seem to show a weak second maximum (e.g. 1210, 1487 and $1634 \mathrm{~m}$ at Byrd). There seems to be no way to achieve such a secondary maximum without recrystallization. Then either recrystallization occurs in some ice undergoing simple shear deep in ice sheets but not in adjacent ice (possibly from differences in ice or from waves of recrystallization), or else recrystallization occurs in all such simple-shear ice but varies in strength relative to rotation. (Or, the observing or contouring conventions chosen by field observers failed to highlight some weak secondary maxima that did exist.)

It thus appears that at least a little recrystallization occurs in deep simple-shear zones of ice sheets, probably along with much polygonization, favoring $n=3$ (see below). This would agree with analyses of ice-sheet shape and velocity that yield $n=3$, because the shear zone near the bed largely controls the ice-sheet shape and velocity (Hamley and others, 1985). However, we cannot absolutely rule out $n<3$. The best data on fabricenhancement factors in simple shear are probably those summarized by Budd and Jacka (1989, fig. 9), so they should be used initially.

\section{Warm, basal multiple fabrics - hypothesis: no good hypothesis}

It is not clear what flow law to use for the very coarse, interlocking grains with a multiple-pole, girdle-type fabric found in warm ice near the bed at Byrd Station (Gow and Williamson, 1976) and elsewhere. The very large grains argue against rapid deformation. The annealing hypothesis of Budd and Jacka (1989; and earlier work) seems to provide the best available explanation. If correct, then this texture and fabric indicate that the ice was deformed rapidly but now is nearly stagnant and at high temperature. When deformation stops, recrystallization in twin orientations is initiated and then "runs away", with grains growing very large. The suggestion of stagnant, or nearly so, ice indicates that there is no need for a flow law for this ice. If a flow law is needed, the girdle fabric shows that recrystallization has occurred, but the very large grains show that recovery processes have been active since the initiation of recrystallization. A recovery-dominated $n=1$ might be slightly more likely, but the evidence is weak.

\section{Ice shelves and ice streams - hypothesis: $\boldsymbol{n}=3$. Fabric effects from Jacka and Maccagnan (1984)}

With ice shelves, and probably with their inland extensions as ice streams, we finally reach a firmer basis for the flow law. Laboratory experiments show clearly that relatively high normal strain rates (high stresses or temperatures) cause girdle-type fabrics (i.e. no $c$-axes near compressional axes), a true steady state of deformation (a constant applied stress yields a constant strain rate, grainsize and $c$-axis fabric, and thus almost certainly a steadystate average dislocation density) and $n=3$ (e.g. Jacka, 1984a, b; Jacka and Maccagnan, 1984). Ice shelves under temperatures and normal stresses approaching laboratory 
values develop girdle-type fabrics and are observed to spread with $n=3$ (e.g. Thomas, 1973; Jezek and others, 1985). Doake and Wolff (1985) argued that ice-shelf spreading is consistent with $n=1$ or $n=3$, but required that other workers significantly underestimated their errors and that the good, high-stress data from Amery Ice Shelf, Antarctica, be ignored to avoid the natural conclusion from the data that $n=3$. A girdle-type fabric has been observed seismically on Ice Stream B in West Antarctica (Blankenship and others, 1989).

The girdle-type fabrics of the ice shelves and ice streams show that recrystallization occurs. Because this requires high stored strain energy in the grain or subgrain into which a new boundary migrates, rather than just in sub-grain boundaries, this shows that grains contain a large dislocation density at least equal to the steady-state value, and thus argues for $n=3$. Then $n=3$ is a good hypothesis wherever recrystallization occurs. (At higher stresses than occur in natural ice sheets, $n>3$ is possible.)

Jacka and Maccagnan (1984) showed how the angle of the girdle fabric varies between onset of recrystallization and approach to steady state, and how the ice hardness depends on this angle. The values recommended by Jacka and Maccagnan provide a good starting point for modeling.

\section{Summary of hypotheses}

We thus sketch a crude picture of macroscopic and microscopic properties in cold natural ice. Rapid deformation under normal stresses in ice shelves and ice streams causes recrystallization and $n=3$. Simple-shear deformation in deep ice probably causes recrystallization and $n=3$, but the evidence is not as strong as for ice shelves and does not rule out $n=1$ or some intermediate value. Polygonization probably dominates in somewhat shallower ice with normal stresses, and recovery processes must be important. The recovery processes suggest $n<3$, including $n=1$, but the evidence is weak. In very shallow ice with normal grain growth, recovery processes are most rapid relative to storage of strain energy, dislocation multiplication may be suppressed, and $n=1$ (or at least $n<3$ ) is most likely, but proof again is lacking. A reasonable approach to testing the flow law would be to divide the ice sheet into layers based on macroscopic observations of fabric and texture, and to assume $n$ has a constant value (or a constant slope of variation) within a layer.

The onset or re-orientation of recrystallization during progressive burial of ice or initiation of streaming flow require that the ice first harden from storage of strain energy and then soften from recrystallization removal of this strain energy, as shown in Figure 3. This should cause recrystallizating zones to be surrounded by bands of stiff ice, which may have important implications for ice flow (which I (Alley, 1990) have discussed briefly and hope to explore in a future manuscript).

Progressive strain causes fabric formation. c-axes rotate toward compressive axes in normal grain growth and polygonization, in a process that cannot obtain a true steady state under normal stresses. $c$-axes rotate to the normal to the shear plane in simple shear, and form a girdle around compressive axes in recrystallization, and can reach a steady state in these cases. The fabric effect on creep rates is a tensor property but reduces to a simple enhancement factor for deformation in one stress state. Laboratory experiments constrain the enhancement factor well for simple shear and recrystallization, and theory constrains the smaller variation in enhancement factor under normal grain growth and polygonization.

\section{ACKNOWLEDGEMENTS}

I thank members of the PIREX workshop, A.J. Gow, T. H. Jacka and W. S. B. Paterson for helpful suggestions. This research was funded in part by the U.S. National Science Foundation under grants DPP-8822027 and DPP-8915995. This is contribution No. GISP2 91-04 of the Greenland Ice Sheet Project Two (GISP2).

\section{REFERENCES}

Alley, R. B. 1987. Firn densification by grain-boundary sliding: a first model. 7. Phys. (Paris), 48, Colloq. C1, 249-254. (Supplément au 3.)

Alley, R. B. 1988. Fabrics in polar ice sheets: development and prediction. Science, 240(4851), 493-495.

Alley, R. B. 1990. Flow-law hypotheses for ice-sheet modeling. [Abstract.] Eos, 71(43), 1308.

Alley, R. B., J.H. Perepezko and C. R. Bentley. 1986a. Grain growth in polar ice: I. Theory. 7. Glaciol., 32(112), 415-424.

Alley, R. B., J.H. Perepezko and C. R. Bentley. 1986b. Grain growth in polar ice: II. Application. J. Glaciol., 32(112), 425-433.

Azuma, N. and A. Higashi. 1985. Formation processes of ice fabric pattern in ice sheets. Ann. Glaciol., 6, 130-134.

Blankenship, D. D., R. B. Alley and C. R. Bentley. 1989. Fabric development in ice sheets: seismic anisotropy. [Abstract.] Eos, 70(15), 462.

Budd, W.F. and T.H. Jacka. 1989. A review of ice rheology for ice sheet modelling. Cold Reg. Sci. Technol., 16(2), 107-144.

Dieter, G.E. 1976. Mechanical metallurgy. Second edition. New York, McGraw-Hill.

Doake, C. S. M. and E. W. Wolff. 1985. Flow law for ice in polar ice sheets. Nature, 314(6008), 255-257.

Duval, P. and L. Lliboutry. 1985. Superplasticity owing to grain growth in polar ices. F. Glaciol., 31(107), 6062.

Duval, P., M. F. Ashby and I. Anderman. 1983. Ratecontrolling processes in the creep of polycrystalline ice. J. Phys. Chem., 87(21), 4066-4074.

Etchecopar, A. 1977. A plane kinematic model of progressive deformation in a polycrystalline aggregate. Tectonophysics, 38, 121-139.

Fujita, S., M. Nakawo and S. Mae. 1987. Orientation of the 700-m Mizuho core and its strain history. In Matsuda, T., ed. Papers presented at the "Ninth Symposium on Polar Meteorology and Glaciology", held at the National Institute of Polar Research, Tokyo, December 11-12, 1986. Tokyo, National Institute of Polar Research, 122-131. (Proceedings of the NIPR Symposium on Polar Meteorology and Glaciology 1.) 
Fukuda, A., T. Hondoh and A. Higashi. 1987. Dislocation mechanisms of plastic deformation of ice. $\mathcal{F}$. Phys. (Paris), 48, Colloq. C1, 163-173. (Supplément au 3.)

Gleiter, H. 1979. Vacancy drag - the generation of vacancies by interface migration. Acta Metall., 27(11), 1749-1754.

Gow, A.J. 1969. On the rates of growth of grains and crystals in south polar firn. 7. Glaciol., 8(53), 241-252.

Gow, A.J. and T. Williamson. 1976. Rheological implications of the internal structure and crystal fabrics of the West Antarctic ice sheet as revealed by deep core drilling at Byrd Station. CRREL Rep. 76-35.

Hamley, T.C., I. N. Smith and N.W. Young. 1985. Mass-balance and ice-flow-law parameters for East Antarctica. 7. Glaciol., 31(109), 334-339.

Herron, S. L. and C. C. Langway, Jr. 1982. A comparison of ice fabrics and textures at Camp Century, Greenland and Byrd Station, Antarctica. Ann. Glaciol., 3, $118-124$.

Herron, S. L., C. C. Langway, Jr and K. A. Brugger. 1985. Ultrasonic velocities and crystalline anisotropy in the ice core from Dye 3, Greenland. In Langway, C. C., Jr, H. Oeschger and W. Dansgaard, eds. Greenland ice core: geophysics, geochemistry and the environment. Washington, DC, American Geophysical Union, 23-31. (Geophys-ical Monograph 33.)

Hobbs, P. V. 1974. Ice physics. Oxford, Clarendon Press.

Hooke, R. LeB. and P.J. Hudleston. 1980. Ice fabrics in a vertical flow plane, Barnes Ice Cap, Canada. 7 . Glaciol., 25(92), 195-214.

Ignat, M. and H.J. Frost. 1987. Grain boundary sliding in ice. 7. Phys. (Paris), 48, Colloq. Cl, 189-195. (Supplément au 3.)

Jacka, T.H. 1984a. Laboratory studies on relationships between ice crystal size and flow rate. Cold Reg. Sci. Technol., 10(1), 31-42.

Jacka, T.H. 1984b. The time and strain required for development of minimum strain rates in ice. Cold Reg. Sci. Technol., 8(3), 261-268.

Jacka, T.H. and Gao Xiangqun. 1989. Ice crystal orientation fabrics and related glaciological parameters from neighbouring Antarctic core sites. In Guo Kun, ed. Proceedings of the International Symposium on Antarctic Research. Beijing, China Ocean Press, 41-52.

Jacka, T.H. and M. Maccagnan. 1984. Ice crystallographic and strain rate changes with strain in compression and extension. Cold Reg. Sci. Technol., 8(3), 269-286.

Jezek, K. C., R. B. Alley and R.H. Thomas. 1985. Rheology of glacier ice. Science, 227(4692), 1335-1337.
Kamb, W. B. 1972. Experimental recrystallization of ice under stress. In Heard, H. C., I. Y. Borg, N. L. Carter and C.B. Raleigh, eds. Flow and fracture of rocks. Washington, DC, American Geophysical Union, 211241. (Geophysical Monograph 16.)

Lipenkov, V.Ya., N.I. Barkov, P. Duval and P. Pimienta. 1989. Crystalline texture of the $2083 \mathrm{~m}$ ice core at Vostok Station, Antarctica. J. Glaciol., 35(121), 392-398.

Lister, G. S., M. S. Paterson and B. E. Hobbs. 1978. The simulation of fabric development in plastic deformation and its application to quartzite: the model. Tectonophysics, 45(2/3), 107-158.

Lliboutry, L. and P. Duval. 1985. Various isotropic and anisotropic ices found in glaciers and polar ice caps and their corresponding rheologies. Annales Geophysicae, 3(2), 207-224.

Matsuda, M. and G. Wakahama. 1978. Crystallographic structure of polycrystalline ice. F. Glaciol., 21(85), 607620.

Paterson, W. S. B. 1985. Flow law for ice in polar ice sheets. Nature, 318(6041), 82-83.

Pimienta, P. and P. Duval. 1987. Rate controlling processes in the creep of polar glacier ice. F. Phys. (Paris), 48, Colloq. C1, 243-248. (Supplément au 3.)

Poirier, J. -P. 1985. Creep of crystals. Cambridge, Cambridge University Press.

Russell-Head, D. S. and W. F. Budd. 1979. Ice-sheet flow properties derived from bore-hole shear measuremerits combined with ice-core studies. 7. Glaciol., 24(90), 117-130.

Thomas, R.H. 1973. The creep of ice shelves: interpretation of observed behaviour. F. Glaciol., 12(64), 55-70.

Verhoeven, J.D. 1975. Fundamentals of physical metallurgy. New York, Wiley and Sons.

Weertman, J. 1968. Dislocation climb theory of steadystate creep. Trans. Am. Soc. Metals, 61(4), 681-694.

Weertman, J. 1973. Creep of ice. In Whalley, E., S. J. Jones and L. W. Gold, eds. Physics and chemistry of ice. Ottawa, Royal Society of Canada, 320-337.

Weertman, J. 1983. Creep deformation of ice. Annu. Rev. Earth Planet. Sci., 11, 215-240.

Weertman, J. 1985. Unsolved problems of creep. Nature, 314(6008), 227.

Weertman, J. and J.R. Weertman. 1964. Elementary dislocation theory. New York, Macmillan; London, Collier-Macmillan.

The accuracy of references in the text and in this list is the responsibility of the author, to whom queries should be addressed. 